\title{
Installing and Using the Penn Forced Aligner (P2FA) Chinese
}

Cong Zhang

If you are reading this article, you probably already know what P2FA is - in brief, it is a tool that you can use to align your transcriptions to your audio files, phoneme by phoneme.

It is not the only existing forced aligner. Kelley and Tucker (2018) has a brief summary on this topic:

"A number of forced aligners already exist. Some rely on hidden Markov models, such as the Penn Forced Aligner [2], the ProsodyLab Aligner [3], MAUS [4], and the Montreal Forced Aligner [1], while Gentle [5] relies on neural networks."

[1] M. McAuliffe, M. Socolof, S. Mihuc, M. Wagner, and M. Sonderegger, "Montreal Forced Aligner: Trainable text-speech alignment using Kaldi," in Interspeech 2017, 2017, pp. 498-502.

[2] J. Yuan and M. Liberman, "Speaker identification on the SCOTUS corpus," Journal of the Acoustical Society of America, vol. 123, no. 5, p. 3878, 2008.

[3] K. Gorman, J. Howell, and M. Wagner, "Prosodylab-aligner: A tool for forced alignment of laboratory speech," Canadian Acoustics, vol. 39, no. 3, pp. 192-193, 2011.

[4] T. Kisler, F. Schiel, and H. Sloetjes, "Signal processing via web services: The use case WebMAUS," in Digital Humanities Conference 2012, 2012, pp. 30-34.

[5] R. M. Ochshorn and M. Hawkins, "Gentle," 2017. [Online]. Available:

https://lowerquality.com/gentle/

I haver also tried Easy Align

(http://latlcui.unige.ch/phonetique/easyalign/easyalign_unpublished.pdf) and a few others, including Praat's own aligner.

To be honest, none of them is accurate. So, please do not skip manual correction even if you have a lot of data.

Five basic steps are involved in installing and using the Mandarin Chinese version of P2FA:

- Installing Cygwin

- Installing htk

- Installing sox

- Installing python

- Downloading the English and the Chinese versions of P2FA

You also need:

Wav sound files (Step 27)

Text files (Step 28) 


\section{Steps:}

1. Install Python https://www.python.org/downloads/ . Version 2.7 is recommended, but I have Python 3 and it works too.

2. Install Cygwin. Under the "Installing Cygwin" section on their homepage, there are choices for a 64-bit installation and a 32-bit installation. Choose the 32-bit one regardless. It has to be 32-bit Cygwin even if your computer is 64-bit. Here's a link (https://cygwin.com/setup-x86.exe ) for the download.

3. Install with the following options (by pressing the little button that has two arrows (3):

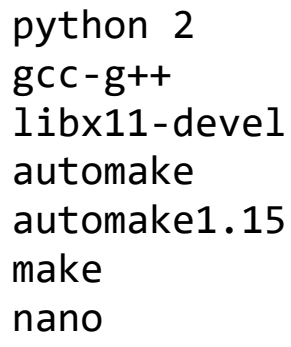

Install it to C: \cygwin folder. I know it makes your drives messy but let's just do this to avoid complications. So, now you have a $C$ : \cygwin folder.

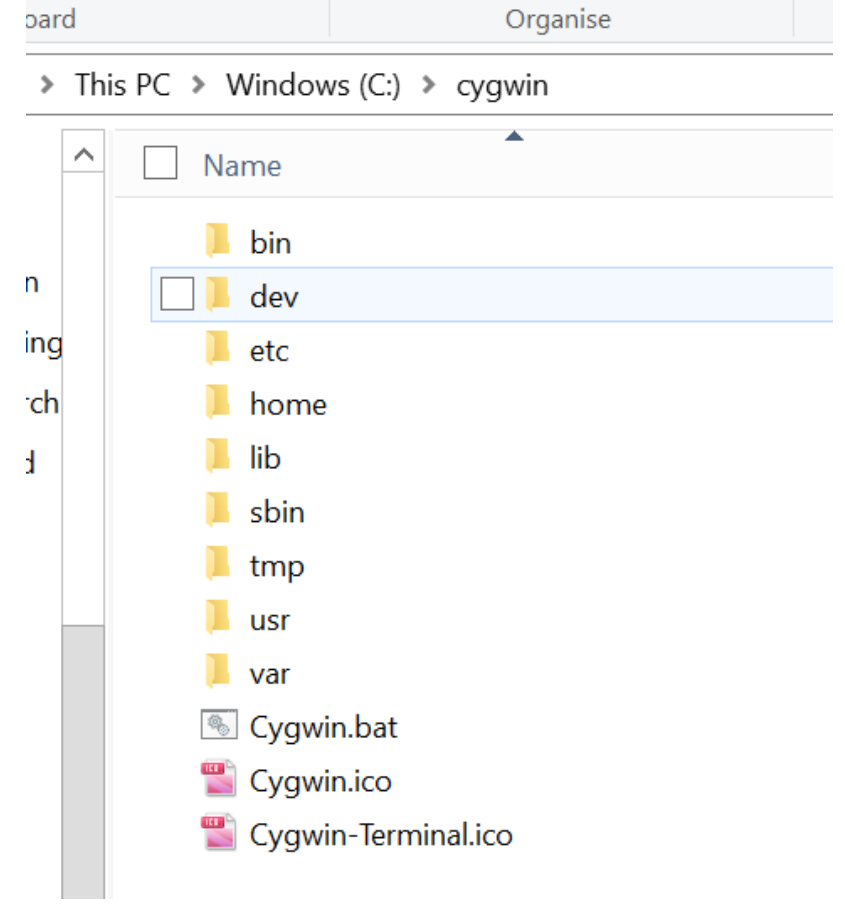

4. Run Cygwin

5. Go to HTK website http://htk.eng.cam.ac.uk/register.shtml and register. Yes, you have to sign up so that you can download. It's free.

6. Download htk-3.4.tar.gz from http://htk.eng.cam.ac.uk/ftp/software/ Note that it has to be htk-3.4 (not 3.41 or anything later). 
7. Create folder in C: (using windows filesystem. Just do it the usual way - nothing fancy about this step) called cygwinfolders. Now you have a folder C: \cygwinfolders

8. Copy the htk file to the newly created folder C: \cygwinfolders.

9. In the Cygwin console (the one with black background), change working directory to $\mathrm{C}$ : \cygwinfolders.

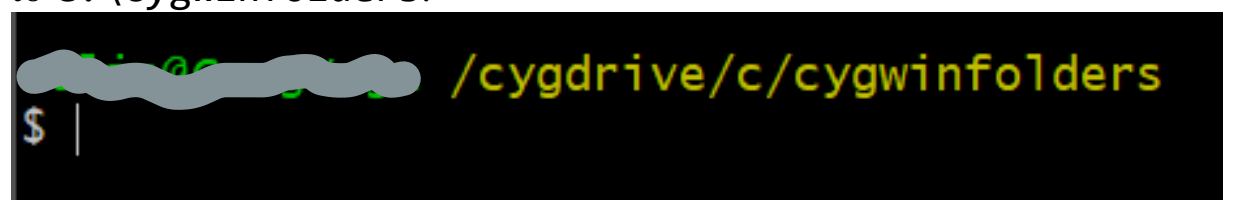

10. In the Cygwin console, type:

tar xzf htk-3.4.tar.gz

Press the Enter key

11. That will create a folder: htk. Now you have C: \cygwinfolders \htk

12. Using windows file explorer:

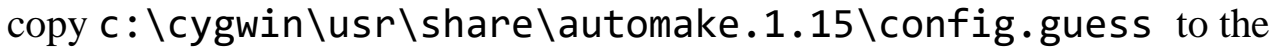

C: \cygwinfolders $\backslash$ htk folder (overwriting the existing file)

13. Change the directory to the htk folder in Cygwin. Type in the Cygwin console:

cd htk

Press the Enter key

./configure

Press the Enter key

14. Expand the width of the Cygwin window by dragging the right-hand edge to the right. In Cygwin console, type:

nano HLMTools/makefile

15. A black text editor box will appear: Go down to mkinstalldir (C:Icygwinfoldersไhtk\HLMTools\Makefile.in): Line 77 has spaces in front of "if" : delete all the spaces, press tab once, and now "if" should have the same indentation as the "for" on line 74.

16. Then press:

ctrl $+x$

$y$

Press the Enter key

And the editor box will close

17. In Cygwin, type:

make all

Press the Enter key

make install

Press the Enter key 
18. Create a folder called exes in cygwinfolders. Now you have C: \cygwinfolders \exes

19. Copy all the .exe files from hlmtools and htktools to the C: \cygwinfolders \exes folder

20. Download and install sox http://sox.sourceforge.net/

21. Find the sox.exe in c: \program files (x86)\sox (whatever version) and copy it to $c: \backslash c y g w i n \backslash b i n \backslash$

22. Edit path in environment variables in system properties - Advanced and add link to sox, to htk folder and cygwindfolders folder. This is how:

- Type environment variables in the windows start box (or Cortana) and press enter when it has found something. (Google "environment variables Windows 10 , if you can't find it.)

- It should then open up the system properties box.

- Click environment variables at the bottom

- In the box that opens look for the 'path' variable and double click it.

- Then add the folders in the same format as you can see: - your sox folder (probably something like C: \program files (x86) \sox14-4-2)

- C: \cygwinfolders

- C: \cygwinfolders $\backslash$ exes

23. Download the aligner for English: https://web.sas.upenn.edu/phoneticslab/files/2016/05/Penn-Phonetics-forced-aligner-2d2jfwb.zip

24. Download the aligner for Chinese:

http://languagelog.ldc.upenn.edu/myl/ChineseAligner.tgz If you unzip it, and get a file called ChineseAligner without any extension, then add the tar extension to it and further unzip until you get Forced_Aligner_Mandarin folder. Move contents in the folder to cp2fa file (see next step).

25. Unzip the English one into a folder called p2fa and the Chinese one a folder called cp2fa. Now we have $C: \backslash c y g w i n f o l d e r s \backslash p 2 f a$ and $C: \backslash c y g w i n f o l d e r s \backslash c p 2 f a$

(Actually, I use ppfa instead of p2fa, just in case numbers cause problems.)

26. Copy align.py from the English version $C$ : \cygwinfolders $\backslash p 2 f a$ to the Chinese version C: \cygwinfolders $\backslash c p 2 f a$ (This is because on my computer, the Chinese version does not create proper TextGrid files. The code seems to be incomplete for some reason. You can try Calign.py in the Chinese version to see whether it works. If you do try, Make sure the homedir in the Calign.py script is changed to cp2fa folder: homedir = 'c:/cygwinfolders/cp2fa' ) 


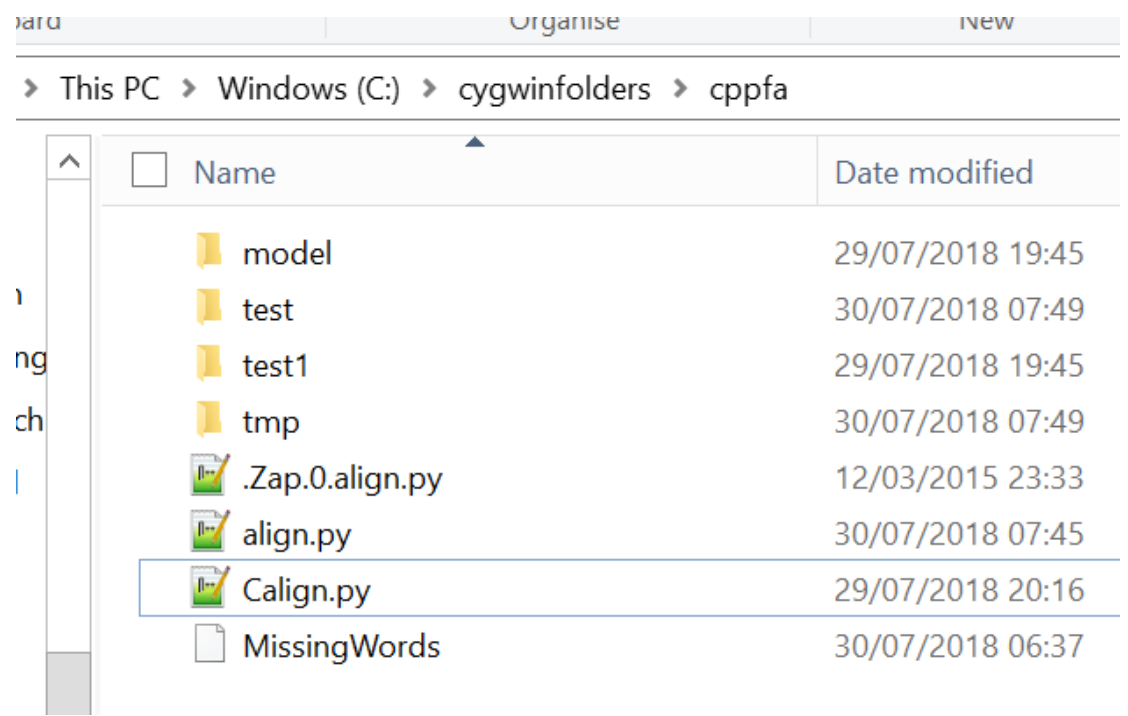

27. Change from 'polyphase' to 'rate' in line 46 of the script "C:Icygwinfolderslcp2falalign.py"

28. In Cygwin, change the working directory to cp2fa folder:

cd C: \cygwinfolders \cp2fa

Press the Enter key

29. In Cygwin, type:

nano align.py

Press the Enter key

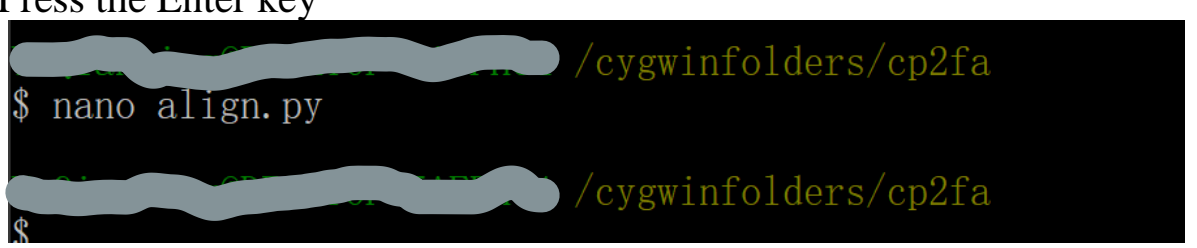

30. Prepare your files: A .wav file and a .txt file. Make sure the wav file is sampled at $8000 \mathrm{~Hz}$ or $16000 \mathrm{~Hz}$. If your sampling rate is neither of these two, your first option is to modify the align.py script:

Edit line 46:

The original looks like this:

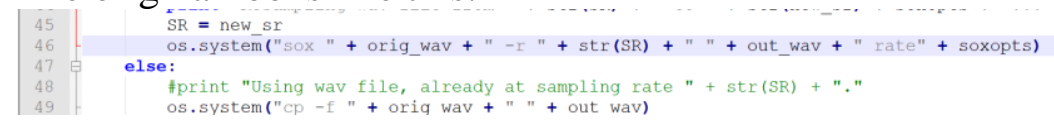

But change Line 46 to the following:

os.system("c:/cygwin/bin/sox.exe " + orig_wav + " -r 8000 " + out_wav + " rate " + soxopts)

The spaces inside the quotation marks are very important. Do not ignore.

The ' 8000 ' here can also be 16000 .

Then, replace the 11025 on line 40 with 8000 or 16000 (keep it consistent with the previous rate), depending on your preference. Note the Chinese version does not have a $11025 \mathrm{~Hz}$ option. Do not change the 11025 occurrences. 
Alternatively, you can also resample your files using sox in the command line:

- At windows start menu, open up cmd (aka Command Prompt)

- You should then be able to enter:

Sox yourfilename.wav $-r 8000$ yourresampledfilename.wav

Or, if these both sound too complicated, just do the resampling in Praat, Audacity, or other software applications that you're comfortable with.

31. The txt file should be your transcription. Make sure the encoding of the txt files is UTF-8. It is a good idea to include an 'sp' at each end of your utterance and have spaces between characters.

32. In Cygwin, type:

python align.py test/test_8000.wav test/ test_8000.txt test/ test_8000.Textgrid

Press the Enter key

The format is "python align.py test/source file.wav textfile.text output

file.Textgrid"

This is an example file in the aligner. You can find the TextGrid file in the C: \cygwinfolders \cp2fa\test folder now.

There are other tutorials online that are very useful. For example,

Will Styler, Using the HTK and the Penn Phonetics Lab Forced Aligner on Mac

OS X, http://wstyler.ucsd.edu/posts/p2fa_mac.html

The one I originally followed was "Using P2FA" by John M. Riebold. It was quite clear but it is not available online anymore.

\section{Acknowledgements:}

This guide is based on the guidance notes that Mr Robert Greenock, our IT officer at University of Oxford, wrote for me. Without his guidance of which versions of the software applications to install, I would be stuck at the very beginning, just like last year, and the year before last year, and the year before the year before last year, and the year before the year before the year before last year... 